\title{
An Efficient Algorithm for Bandpass Sampling of Multiple RF Signals
}

\author{
Junghwa Bae and Jinwoo Park
}

\begin{abstract}
This letter proposes, based on a bandpass sampling theory, a novel method to find available sampling ranges with a low computational complexity and high accuracy for multiple bandpass radio frequency signals. Guard-bands between downconverted signal spectrums are also taken into consideration in determining a minimum sampling frequency. We verify its validity through simulations in terms of the sampling ranges, the minimum sampling frequency, and computational efficiency.
\end{abstract}

Index Terms-Bandpass sampling, software-defined ratio (SDR), sub-sampling.

\section{INTRODUCTION}

B ANDPASS sampling is a method that downconverts analog bandpass signals to baseband or low intermediate frequency (low-IF) digital signals without analog mixers [1]. This technique, called the first-order bandpass sampling or sub-sampling, has therefore been considered as a core element for a software-defined radio (SDR).

A basic principle for downconversion of multiple radio frequency (RF) signals using the bandpass sampling has been introduced in [2]. In [3], they have described schemes to determine valid bandpass sampling frequencies for two distinct RF signals. A generalized solution for downconversion of multiple RF signals has recently been proposed in [4]. It is, however, noted in [4] that a constraint in finding the sampling ranges is suggested such that each replica of the RF signal is placed at each segment of the sampled bandwidth divided proportional to the size of the signal's bandwidth, which may lead to a failure in finding all possible sampling ranges. Furthermore, most of the previous works have not taken into consideration a guard-band or marginal spacing between downconverted signals when finding the sampling frequency for downconverting the multiple RF signals. Such a guard-band can mitigate strict requirements of practical implementation of the bandpass sampling and lessen in part adverse effects from inaccuracy involved in the choices of components and system design, such as aliasing effect due to sampling clock jitter and carrier frequency instability [5], and adjacent channel interferences.

In this letter, we propose an algorithm for finding valid sampling ranges and a minimum sampling rate more accurately and

Manuscript received July 10, 2005; revised October 28, 2005. This work was supported by the University IT Research Center Project at Korea University. The associate editor coordinating the review of this manuscript and approving it for publication was Dr. Gerald Matz.

The authors are with the Department of Electronics Engineering, Korea University, 136-701 Seoul, Korea (e-mail: iruntop@korea.ac.kr; jwpark@korea.ac.kr).

Digital Object Identifier 10.1109/LSP.2005.863694 successfully with a low complexity in computation as well as with a suitable consideration of the guard-band.

\section{Proposed Method for Bandpass SAMPLing WITH GUARD-BAND}

Assume that $N$ multiple bandpass signals $f_{k}(t), k=$ $1,2, \cdots, N$ are given for downconversion. Let $f_{S}, f_{C_{k}}, f_{U_{k}}$, $f_{L_{k}}, f_{I F_{k}}$, and $B W_{k}$ denote sampling frequency, carrier frequency, upper cutoff frequency, lower cutoff frequency, IF, and information bandwidth of $f_{k}(t)$, respectively. We also assume that $f_{U_{k}}=f_{C_{k}}+\left(B W_{k} / 2\right), f_{L_{k}}=f_{C_{k}}-\left(B W_{k} / 2\right)$, and $f_{C_{i}}<f_{C_{i+1}}$ for $i=1,2, \cdots, N-1$, and the spectrum of an $\mathrm{RF}$ bandpass signal is assumed to be band-limited as follows:

$$
\left|F_{k}(f)\right|=0, \text { for }|f| \geq f_{U_{k}} \text { and }|f| \leq f_{L_{k}}, k=1,2, \cdots, N
$$

where $F_{k}(f)$ denotes the spectrum of $f_{k}(t)$. This assumption may act as a strict requirement in the practical system design. Achievements of the supporting technologies, such as highly selective bandpass filters, a high-speed A/D converter with much low aperture jitter, advanced RF chip technologies, and DSP chips, have been widely made in the various areas to fulfill such requirements related to the hardware implementation [6].

In a sampled bandwidth $\left[0, f_{S} / 2\right]$, the total number of possible permutations of signal placements or replica orderings is $N ! \times 2^{N}$, so that such a large number of permutations may rather cause a difficulty in finding valid sampling ranges. We thus limit our interest to one particular case, a signal permutation of normal placement and no change in the ordering of the given RF signals, namely, $f_{I F_{i}}<f_{I F_{i+1}}, i=1,2, \cdots, N-1$, as assumed in [4]. Fig. 1 illustrates the signal spectrum obtained by the bandpass sampling, which consists of the spectrum of the $N$ RF signals and those replicas. Also note that the guard-bands between the downconverted signals appear arbitrarily because they can be determined only after placing the RF signal spectrums in the sampled bandwidth with a properly chosen sampling frequency. To reduce such ambiguity about the guard-band, we specify a minimum guard-band $G B_{\min }(\mathrm{Hz})$ between the adjacent downconverted signals in the sampled bandwidth, as shown in Fig. 1.

To find feasible bandpass sampling ranges below the Nyquist rate, two basic constraints must be satisfied: a boundary constraint in the sampled bandwidth and a nonoverlapping constraint between adjacent IF signals. The boundary constraint means that the replicas of two signals $F_{1}(f)$ and $F_{N}(f)$ should be positioned within the sampled bandwidth so that aliasing by the negative frequency part of each signal should not occur 


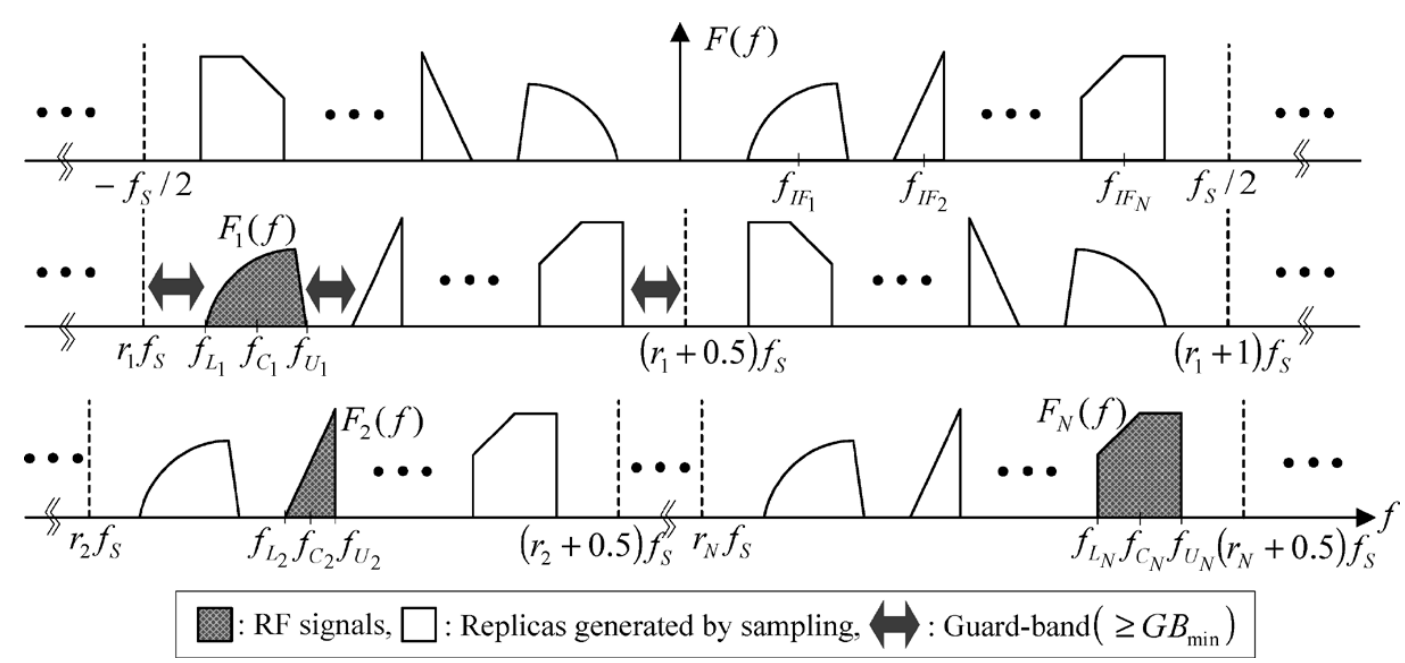

Fig. 1. Spectrum of multiple RF signals after bandpass sampling.

at both boundaries, i.e., 0 and $f_{S} / 2 \mathrm{~Hz}$, in the sampled bandwidth. Taking account of the minimum guard-band $G B_{\min }$ in the sampling range, we can obtain the following equations by the boundary constraint:

$$
r_{1} f_{S} \leq\left(f_{L_{1}}-G B_{\min }\right)
$$

and

$$
\left(r_{N}+0.5\right) f_{S} \geq\left(f_{U_{N}}+G B_{\min }\right)
$$

where $r_{k}$ is defined by $r_{k}=\left\lfloor\left(f_{L_{k}}-G B_{\min }\right) / f_{S}\right\rfloor$ for $k=$ $1,2, \cdots, N$ as a frequency shift coefficient with the guard-band, which is similar to $m_{i}$ in [4]. Here, \lfloor\rfloor denotes a floor function. Combining (2) and (3), we can find an intersection range as

$$
\frac{f_{U_{N}}+G B_{\min }}{r_{N}+0.5} \leq f_{S} \leq \frac{f_{L_{1}}-G B_{\min }}{r_{1}} .
$$

The range of each $r_{k}$ now becomes

$$
\begin{aligned}
1 & \leq r_{k} \\
& \leq\left\lfloor\frac{f_{L_{k}}-G B_{\min }}{2 \cdot\left\{B W_{1}+B W_{2}+\cdots+B W_{N}+(N+1) \cdot G B_{\min }\right\}}\right\rfloor .
\end{aligned}
$$

Note that the range of $r_{2}, r_{3}, \cdots, r_{N}$ except $r_{1}$ can be considerably reduced as explained below. The range of $f_{L_{1}}-G B_{\min }$ can be found as

$$
r_{1} f_{S}<\left(f_{L_{1}}-G B_{\min }\right)<\left(r_{1}+0.5\right) f_{S} .
$$

Here let us define $a_{k}=\left(f_{L_{k}}-G B_{\min }\right) /\left(f_{L_{1}}-G B_{\min }\right)$. By multiplying (6) by $a_{k} / f_{S}$ and taking \lfloor\rfloor , we can obtain the ranges of $r_{2}, r_{3}, \cdots, r_{N}$ as

$$
\left\lfloor a_{k} r_{1}\right\rfloor \leq r_{k} \leq\left\lfloor a_{k}\left(r_{1}+0.5\right)\right\rfloor \text { for } r_{2}, r_{3}, \cdots, r_{N} .
$$

This implies that once $r_{1}$ is found, the range for each of $r_{2}, r_{3}, \cdots, r_{N}$ can be confined to a certain limited range by (7). In addition, the range of each $r_{2}, r_{3}, \cdots, r_{N}$ is relatively small because $a_{k}$ is generally small. Therefore, the iteration or loop number required in searching the feasible sampling ranges can be considerably reduced, compared to the method in [4].
It is also noted that the larger the minimum guard-band is set, the smaller the maximum value of $r_{1}$ is and the shorter the calculation time we need for finding the sampling ranges.

We now consider another constraint that IF signals in the sampled bandwidth should not overlap each other. Avoidance of overlapping between replicas of $F_{i}(f)$ and $F_{i+1}(f)$ for $i=$ $1,2, \cdots, N-1$ can be expressed as

$$
f_{U_{i}}-r_{i} f_{S} \leq\left(f_{L_{i+1}}-G B_{\min }\right)-r_{i+1} f_{S} .
$$

This equation is rewritten as

$$
f_{S} \leq \frac{f_{L_{i+1}}-f_{U_{i}}-G B_{\min }}{r_{i+1}-r_{i}} .
$$

Consequently, the valid sampling ranges for $N$ RF signals are the common ranges satisfying simultaneously (4) and (9), which can be expressed as

$$
\begin{aligned}
& \frac{f_{U_{N}}+G B_{\min }}{r_{N}+0.5} \\
& \quad \leq f_{S} \\
& \quad \leq \min \left\{f_{U B_{1}}, f_{U B_{1,2}}, f_{U B_{2,3}}, \cdots, f_{U B_{N-1, N}}\right\}
\end{aligned}
$$

where $f_{U B_{1}}=\left(f_{L_{1}}-G B_{\min }\right) / r_{1}$ and $f_{U B_{i, i+1}}=\left(f_{L_{i+1}}-\right.$ $\left.f_{U_{i}}-G B_{\min }\right) /\left(r_{i+1}-r_{i}\right)$. Here, in order to obtain the sampling ranges from (10), we need sets of the valid frequency shift coefficient $\left(r_{1}, r_{2}, \cdots, r_{N}\right)$. A condition for the valid coefficient sets can thus be given by

$\frac{f_{U_{N}}+G B_{\min }}{r_{N}+0.5} \leq \min \left\{f_{U B_{1}}, f_{U B_{1,2}}, f_{U B_{2,3}}, \cdots, f_{U B_{N-1, N}}\right\}$.

Once we find the valid coefficient sets satisfying (11) through an iteration process within the given ranges of $r_{k}$, we can obtain, from (10), the possible valid sampling ranges supporting that the size of all the guard-bands are larger than $G B_{\min }(\mathrm{Hz})$. Also note that, since each of the valid coefficient sets found above represent parameters for a valid sampling range, we can calculate the total valid sampling range by summing up all the valid sampling ranges found. In summary, the procedure described above for finding the sampling frequency ranges only requires calculation of (10) with the ranges confined by (7), not 


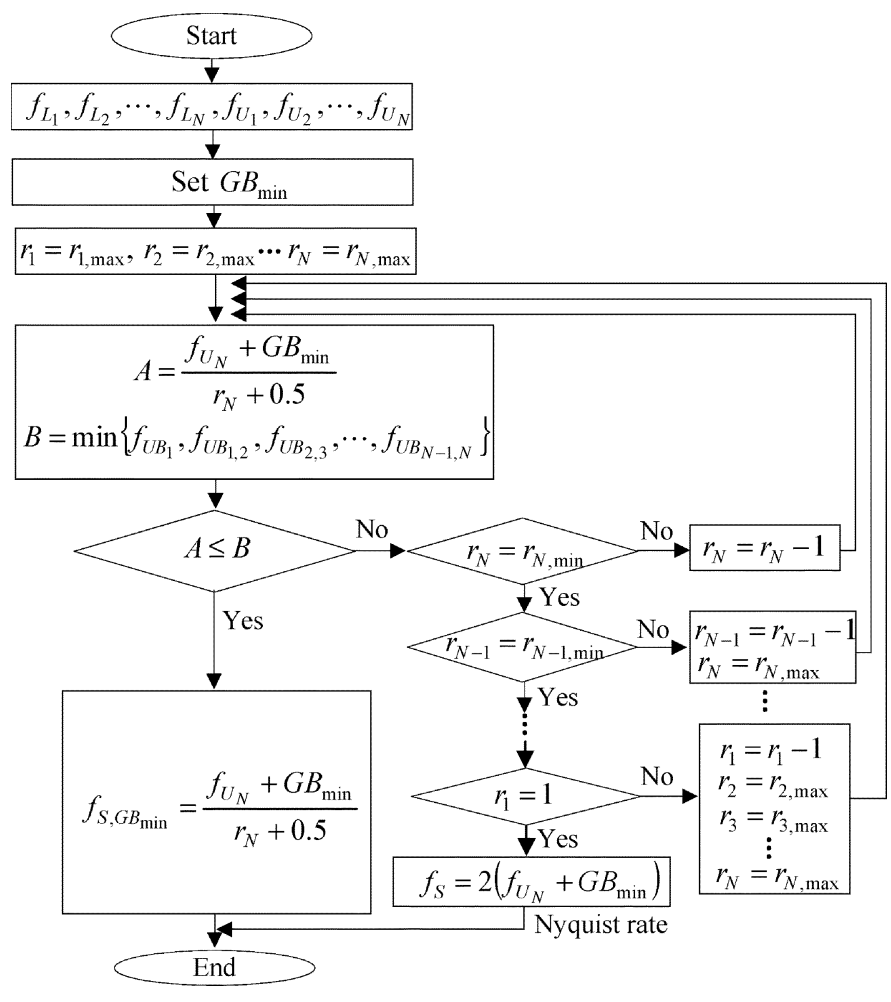

Fig. 2. Algorithm for finding a minimum sampling frequency supporting $G B_{\min }$.

demanding the joint intersection ranges among all the sampling ranges with respect to all $m_{i}$ of each RF signal, as described in [4].

Furthermore, among the valid coefficient sets, the set that has the largest valued-elements provides a minimum sampling frequency for $G B_{\min }$ as follows:

$$
f_{S, G B_{\min }}=\frac{f_{U_{N}}+G B_{\min }}{r_{N}+0.5} .
$$

The procedural flowchart for obtaining this minimum sampling frequency is depicted in Fig. 2, which includes a step of searching the largest valid set in a descending order from the upper bounds of $r_{k}$. This result reveals another advantage of the proposed method because the minimum sampling frequency supporting $G B_{\min }$ can be found by using only one parameter $r_{N}$, resulting in greatly reduced computation time with less iteration number, as shown in Fig. 2. We also note that the result of (12) is, as inferred from (3), the minimum sampling frequency so that the guard-band between $F_{N}(f)$ and $f_{S} / 2$ becomes $G B_{\min }$.

With the calculated $f_{S, G B_{\text {min }}}$, the IFs are found by

$$
f_{I F_{k}}=f_{C_{k}}-r_{k} f_{S, G B_{\min }}
$$

\section{APPLICATIONS AND COMPARISONS}

We first consider a mobile receiver that is supposed to support three RF signals with the same bandwidth such as $f_{C_{1}}=$ 864.3 MHz, $f_{C_{2}}=890.3 \mathrm{MHz}$, and $f_{C_{3}}=935.7 \mathrm{MHz}$ with $B W=200 \mathrm{kHz}$. For comparison with the method in [4], the
TABLE I

COMPUTATION RESUlTS OF BANDPASS SAMPLING FOR THREE RF SigNALS With THE SAME BANDWIDTH

\begin{tabular}{cccc}
\hline \hline & & \multicolumn{2}{c}{ Proposed Method } \\
\cline { 3 - 4 } & & $G B_{\text {min }}=0 \mathrm{~Hz}$ & $G B_{\text {min }}=200 \mathrm{kHz}$ \\
\hline & & $1 \leq r_{1} \leq 720$ & $1 \leq r_{1} \leq 308$ \\
& & $r_{1}=720$ & $r_{1}=308$ \\
Frequency shift & $1 \leq m_{1} \leq 720$ & $741 \leq r_{2} \leq 742$ & $r_{2}=317$ \\
coefficients & $1 \leq m_{2} \leq 741$ & $779 \leq r_{3} \leq 780$ & $r_{3}=333$ \\
& $1 \leq m_{3} \leq 779$ & $r_{1}=719$ & $r_{1}=307$ \\
& & $740 \leq r_{2} \leq 741$ & $r_{2}=316$ \\
& & $r_{3}=778$ & $r_{3}=332$ \\
& & $\vdots$ & $\vdots$ \\
\hline $\begin{array}{c}\text { The number of valid } \\
\text { sampling range }\end{array}$ & 23 & 38 & 20 \\
(Min. iteration & $(330625)$ & $(1670)$ & $(715)$ \\
number) & & & \\
\hline Min. sampling & $2.1390 \mathrm{MHz}$ & $1.6093 \mathrm{MHz}$ & $3.2 \mathrm{MHz}$ \\
frequency with the & $m_{1}=404$ & $r_{1}=537$ & $r_{1}=270$ \\
coefficient set & $m_{2}=416$ & $r_{2}=553$ & $r_{2}=278$ \\
(Min. iteration & $m_{3}=437$ & $r_{3}=581$ & $r_{3}=292$ \\
number) & $(57268)$ & $(422)$ & $(89)$ \\
\hline \hline
\end{tabular}

complexity of the algorithms is examined in terms of the minimum iteration number required in finding the valid sampling ranges and the minimum sampling frequency. The results are summarized in Table I. The minimum iteration number of the proposed method is remarkably reduced to be 1670 , compared with 330625 by the method in [4], because the ranges of $r_{2}$ and $r_{3}$ are confined by $r_{1}$. If we are allowed to adopt a larger $G B_{\min }$, the iteration number can be more reduced. More advantageously, the minimum sampling frequency found by the proposed method is much less, probably providing a greater benefit of a lower frequency operation to system designers in practical implementation, and wider sampling ranges are also provided. It is because the method in [4] presumes a constraint that each IF signal is supposed to place at each per-determined frequency band divided by the weight factors proportional to the size of the signal's bandwidth. Consequently, the sampling ranges found by the method in [4] become a subset of the sampling ranges by the proposed method. What is better with the proposed method is that it can find the valid sampling range in the cases where the method in [4] does not work properly, as shown in the next example.

We now consider another example of a mobile device receiving three standards with the different bandwidth such as a channel of GSM-900 with $f_{C_{1}}=940.1 \mathrm{MHz}$ and $B W_{1}=$ $200 \mathrm{kHz}$, a channel of DAB (Eureka-147 L-Band) with $f_{C_{2}}=$ $1473.054 \mathrm{MHz}$ and $B W_{2}=1.536 \mathrm{MHz}$, and a channel of WLAN IEEE 802.11g with $f_{C_{3}}=2422 \mathrm{MHz}$ and $B W_{3}=$ $20 \mathrm{MHz}$, as shown in Fig. 3(a). First, using the method in [4], we obtain three weight factors of $W_{1}=9.2^{-3}, W_{2}=0.08$, and $W_{3}=1$ for each frequency band. However, valid sampling frequencies using these weight factors cannot be found because the space for placing $F_{1}(f)$, i.e., $\left[0,9.2^{-3} \cdot\left(f_{S} / 2\right)\right]$, is not sufficient in the downconverted spectrum due to a large difference of the signal bandwidths. In such a case, they thus have to adopt other signal permutations as a possible solution. On the other 


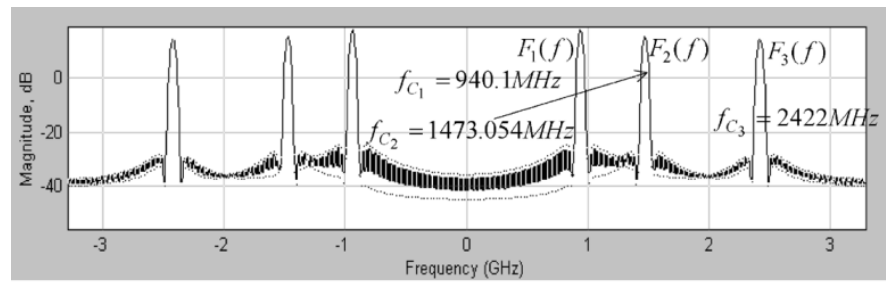

(a)

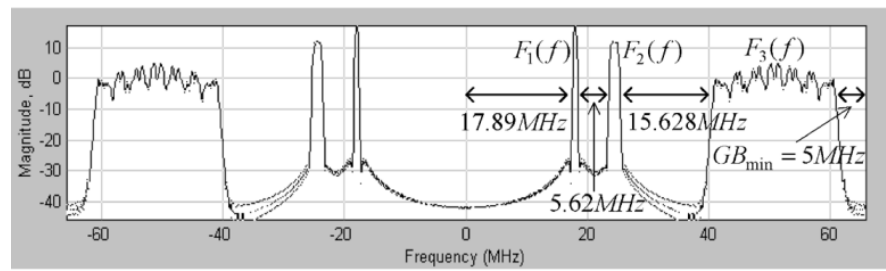

(b)

Fig. 3. (a) Spectrum of three RF signals. (b) IF signals sampled by $f_{S, G B_{\min }}=131.7297 \mathrm{MHz}\left(G B_{\min }=5 \mathrm{MHz}\right)$.

hand, five valid sampling regions for $G B_{\min }=0 \mathrm{~Hz}$ are found by using (10), and the minimum sampling frequency 58.6024 MHz with $r_{1}=16, r_{2}=25$, and $r_{3}=41$ can also be obtained. Furthermore, taking account of $G B_{\min }=5 \mathrm{MHz}$, we can find the minimum sampling frequency $f_{S, G B_{\min }}=131.7297 \mathrm{MHz}$ with $r_{1}=7, r_{2}=11$, and $r_{3}=18$. The iteration number required is only 18 by using the method in Fig. 2. Fig. 3(b) shows successfully downconverted signals illustrating the minimum guard-bands of $5 \mathrm{MHz}$ between $F_{3}(f)$ and $f_{S} / 2$.

\section{CONCLUSION}

In this letter, we have proposed a novel algorithm for finding the valid sampling ranges under the assumption of one permutation of signal placements, especially taking into consideration the minimum guard-band. We also verified through the simulations that the proposed method is superior to the pre-reported work in computational complexity and efficiency for downconversion of multiple RF signals.

\section{REFERENCES}

[1] R. G. Vaughan, N. L. Scott, and D. R. White, "The theory of bandpass sampling," IEEE Trans. Signal Process., vol. 39, no. 9, pp. 1973-1983, Sep. 1991.

[2] D. M. Akos, M. Stockmaster, J. B. Y. Tsui, and J. Caschera, "Direct bandpass sampling of multiple distinct RF signals," IEEE Trans. Commun., vol. 47, no. 7, pp. 983-988, Jul. 1999.

[3] C. H. Tseng and S. C. Chou, "Direct downconversion of multiple RF signals using bandpass sampling," in Proc. ICC, vol. 3, May 2003, pp. 2003-2007.

[4] M. Choe and K. Kim, "Bandpass sampling algorithm with normal and inverse placements for multiple RF signals," IEICE Trans. Commun., vol. E88, no. 2, pp. 754-757, Feb. 2005.

[5] R. Qi, F. P. Coakley, and F. G. Evans, "Practical consideration for bandpass sampling," Electron. Lett., vol. 32, no. 20, pp. 1861-1862, Sep. 1996.

[6] W. Tuttlebee, Software Defined Radio: Enabling Technologies. New York: Wiley, 2002. 